\title{
Intrinsic and Extrinsic Neuronal Mechanisms in Temporal Coding: A Further Look at Neuronal Oscillations
}

\author{
Rémy Lestienne \\ Institut des Neurosciences (CNRS UMR 7624), \\ Université Paris VI, 9 quai St-Bernard, F-75005 PARIS, France
}

\begin{abstract}
Many studies in recent years have been devoted to the detection of fast oscillations in the Central Nervous System (CNS), interpreting them as synchronizing devices. We should, however, refrain from associating too closely the two concepts of synchronization and oscillation. Whereas synchronization is a relatively well-defined concept, by contrast oscillation of a population of neurones in the CNS looks loosely defined, in the sense that both its frequency sharpness and the duration of the oscillatory episodes vary widely from case to case. Also, the functions of oscillations in the brain are multiple and are not confined to synchronization. The paradigmatic instantiation of oscillation in physics is given by the harmonic oscillator, a device particularly suited to tell the time, as in clocks. We will thus examine first the case of oscillations or cycling discharges of neurones, which provide a clock or impose a "tempo" for various kinds of information processing. Neuronal oscillators are rarely just clocks clicking at a fixed frequency. Instead, their frequency is often adjustable and controllable, as in the example of the "chattering cells" discovered in the superficial layers of the visual cortex. Moreover, adjustable frequency oscillators are suitable for use in "phase locked loops" (PLL) networks, a device that can convert time coding to frequency coding; such PLL units have been found in the somatosensory cortex of guinea pigs. Finally, are oscillations stricto sensu necessary to induce synchronization in the discharges of downstream neurones? We know that this is
\end{abstract}

not the case, at least not for local populations of neurones. As a contribution to this question, we propose that repeating patterns in neuronal discharges production may be looked at as one such alternative solution in relation to the processing of information. We review here the case of precisely repeating triplets, detected in the discharges of olfactory mitral cells of a freely breathing rat under odor stimulation.

\section{INTRODUCTION}

In the recent past, fast oscillations in the CNS have been examined as probably aimed at ensuring the synchronicity of discharges in a population of neurones, as seems to have been proposed as early as 1919 by Delage (1919), but revitalized a decade ago by several groups of German researchers (Eckhorn et al, 1988; Gray \& Singer, 1989). Several reviews of arguments for this functional interpretation of oscillations have been recently published (see Singer, 1993; Singer \& Gray, 1995). Singer's arguments on the latter. refer to numerous experiments and observations (Roelfsema et al., 1997) on the visual system of cats and monkeys performed by his group. Note that synchronous oscillations are not limited to the visual system nor to non-human animals. Such oscillations have long been found also, for instance, in the auditory system (Makela \& Hari, 1987; Joliot et al., 1994). Others have observed gamma-band oscillations, apparently correlated to visual recognition tasks in humans (TallonBaudry et al., 1997). One of the best examples of this function of synchronization is given by the olfactory system. It was recently demonstrated that oscillations in the olfactory bulb, or in its 
more or less homologous structure in insects (the antennal lobe),

(a) induce the synchronization of discharges among a population of cells,

(b) are correlated to fine odor discrimination, and

(c) are disrupted when blocking GABA IPSPs $_{A}$ from local inhibitory neurones by means of local injection of picrotoxin (Wehr \& Laurent, 1996; MacLeod \& Laurent, 1996; Stopfer et al., 1997).

Figure 1 displays one of the recent experiments performed by Laurent and his colleagues

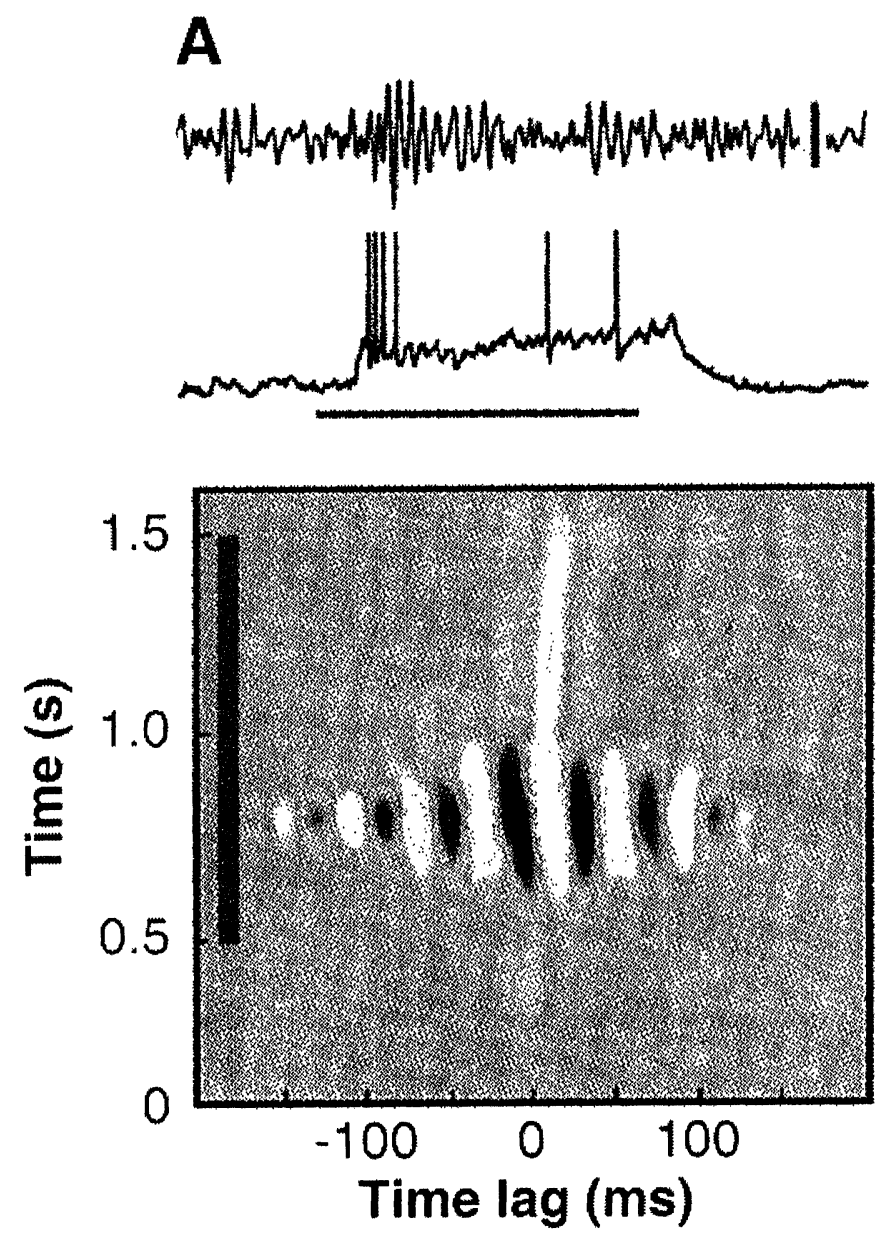

B
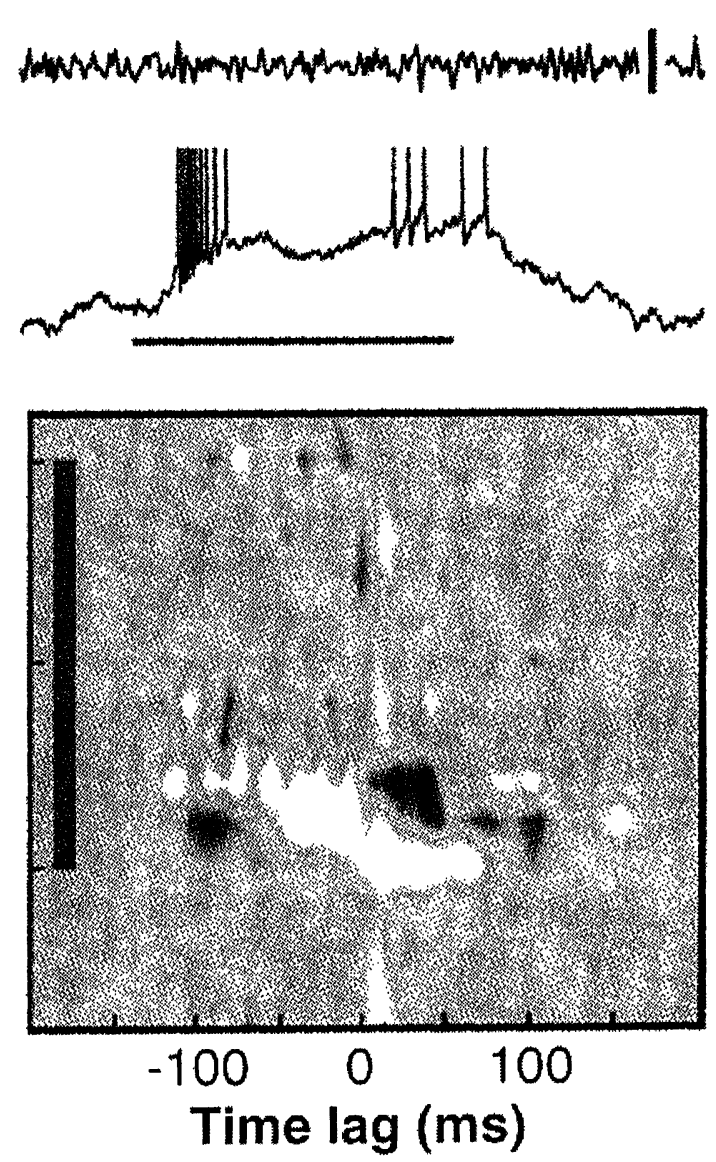

Fig. 1: Picrotoxin injection in the antennal lobe selectively abolishes the oscillatory synchronization but not the responsiveness of Principal Neurones (PN). Top traces: LFP from mushroom body. Middle traces: simultaneous intracellular recording from antennal lobe PN during odor puff (indicated by horizontal bar). Bottom: sliding cross-correlation between LFP and PN traces. Only the relative (and not the absolute) values of the cross-correlation functions matter. A: Control response to mint odor. The biphasic PN response and the prominent IPSPs are apparent during the odor response. The oscillatory LFP indicates synchronized and rhythmic firing of many other PNs during the odor response. The crosscorrelation between PN and LFP shows a striped pattern with $\sim 50 \mathrm{~ms}$ period during the first half of the odor puff (vertical bar between 0.5 and $1.5 \mathrm{~s}$ ), indicating an odor-evoked transient synchronization between this PN and the LFP. B: same pair as in A, 2 min after pressure injection of $800 \mu \mathrm{M}$ of picrotoxin into the antennal lobe. Although the response pattern of the PN to mint is not qualitatively altered (the period of initial excitation, suppression, and subsequent excitation are preserved), the IPSPs have disappeared (indicating the block of LN mediated fast inhibition ), and the $20 \mathrm{~Hz}$ LFP oscillation are abolished. The cross-correlation is aperiodic (Modified from MacLeod and Laurent, 1996). 
(1996). Intracellular recordings of single units in the antennal lobe of a locust, which play a similar role to the mitral cells in mammals, are recorded simultaneously with the local field potentials (LFP) of the mushroom body, the next downstream structure in the insect olfactory system. Cross-correlations between the two records in successive sliding windows of time are presented in grey shades at left (panel A), showing a clear train of oscillations during a limited period of the odor presentation. The brevity of oscillatory episodes should be noted: they extend for about 200-250 ms and do not last for the whole time span of the odor puff (one second). A close examination of the records shows that the spikes from the antennal lobe principal cell, visible in the intracellular recording shown above the correlogram in panel $\mathrm{A}$, are emitted in phase with the LFP oscillations, measured in the mushroom body and displayed in the upper track in the figure. Injection of picrotoxin at the antennal-lobe level abolishes oscillations in both the antennal lobe and the mushroom body, but does not change the general time course of the principal neurone discharges.

Let us return to the observation that oscillations occupy only a limited part of the time of odor presentation; the same is true of much (but not all) evidence presented so far about coherent oscillations. Correlated oscillations usually range from $50-250 \mathrm{~ms}$ at maximum and occur usually (in the human somato-sensory cortex) at no more than 1-2 episodes per second (Menon et al., 1996). In local populations of neurones, whereas synchronization, measured by the relative height of the central peak of cross correlograms, is strengthened by the appearance of gamma-oscillations, it is already present from the beginning of the response and usually precedes oscillation by several tens of milliseconds (Herculano-Houzel, 1999).

We shall not discuss in more detail the relation between synchronization and oscillation because this subject has been extensively covered elsewhere (Delage, 1919; Eckhorn et al, 1988; Lestienne, 1999). Instead, we should like to enlarge the view and develop the idea that oscillations in the CNS are not confined to the role of a synchronizing device. Conversely, we will look for mechanisms, distinct from oscillations, that might be suited to induce synchronization in local populations of interconnected neurones.

\section{OSCILLATIONS AS CLOCKS OR "TEMPO" PROVIDERS}

Imposing a rhythm in the processing of neuronal information does not necessarily require an internal clock, provided by a neuronal oscillator, if the external stimulation in itself already contains a cycling or recurring component. This is obviously the case with sounds, for which oscillatory components are readily available in the signal itself. An example of such a processing mechanism is given by the use of auditory frequencies in the detection of interaural delays by the owl.

Figure 2 emphasizes some crucial aspects of the problem of detecting the azimuthal direction of a prey by the barn owl. To be able to locate the direction of the former to within 2 degrees of angle, as it does, the owl should discriminate interaural delays of the order of $5 \mu \mathrm{s}$ only (Moiseff \& Konishi, 1981). Discrimination with such precision requires that the neurones of the nucleus laminaris, receiving inputs from both the right and left ears, behave as sharp coincidence detectors, rather than as integrate-and-fire neurones. Such discrimination also requires that the relative phases of the inputs (with respect to the sound signal at the particular frequency channel to which the afferent fiber is tuned) be preserved in the terminal arborization and dendritic tree of the neurones. It is the merit of Gerstner et al. (1996) to have proposed plausible mechanisms for preserving the submillisecond timing accuracy of the phase of spikes in these processes. One might object that the ability of bat to discriminate interaural delays with a precision of $5 \mu \mathrm{s}$ does not imply that any single cell of the nucleus laminaris does so with such precision: Does not the law of large numbers suggest that a gain of precision may be achieved through a collective measurement by a population of cells? 
The answer to this question is that the improvement in precision that is gained by simultaneous measurements in an assembly of neurones is limited because the signals impinging on the various neurones of the assembly are not statistically independent (Briten et al., 1992). Accordingly, the maximum achievable gain in precision, due to collective measurements, can hardly reach a factor of 5. Thus, an accuracy of the order of $25 \mu$ s at each single nucleus laminaris cell would be enough to fulfil the requirements for behavioral discrimination at the level of $5 \mu \mathrm{s}$. The left side of Fig. 2 illustrates the principle of interaural detection by means of a field of coincidence detectors. The nucleus laminaris implements this scheme almost exactly, first imagined on theoretical grounds by Jeffress (1948). The electrotonic distances for a signal to proceed through the terminal arborization should, from purely geometric and conduction velocity considerations, provoke a spreading of inputs of the order of $250 \mu \mathrm{s}$ all over a sweep (right side of Fig. 2, upper panel). The selection by a Hebbian- like mechanism of synapses precisely tuned for a given phase of the external (sound component) frequency would allow the reduction of this spreading to a dispersion of only $25 \mu$ s (right side of Fig. 2, lower panels).

In the interaural delay detection problem, the clock used by the brain comes from the sensory data themselves (the oscillatory nature of the sounds). Inside the brain, neuronal clocks seem to exist as well, particularly for processing sensory information other than sound. In an olfactory bulb under odor stimulation, for instance, the electrocorticogram, or the local field potential, undergoes oscillations (as is well known, this system was the first in which $\gamma$-like oscillations were detected). In the example of the odor-evoked discharges of principal neurones of the antennal lobe of locusts, such discharges are modulated by $\gamma$-like oscillations, which seem to be generated through local inhibitory loops [because those oscillations are disrupted by local micro-injections of picrotoxin (MacLeod \& Laurent, 1996)]. The so-called chattering cells, discovered in the super-
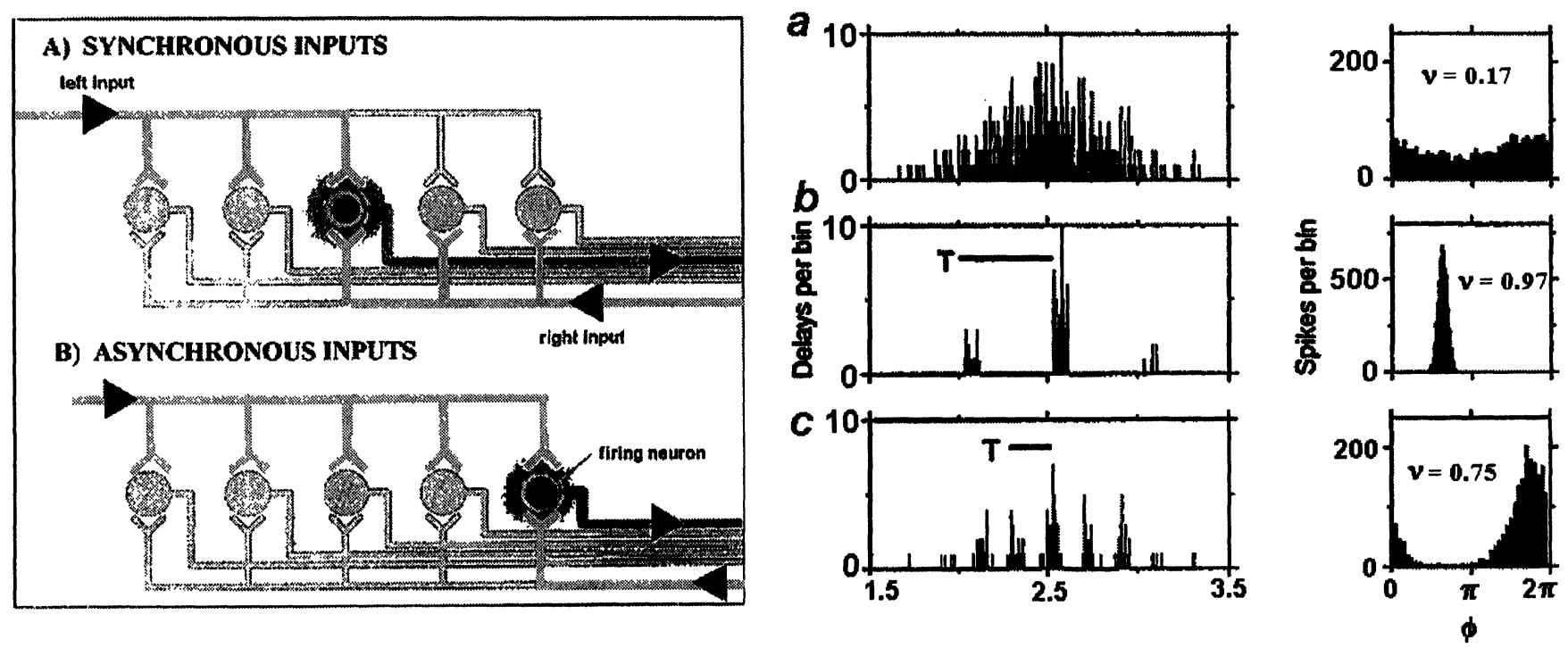

Fig. 2: Detecting submillisecond coincidences in the nucleus laminaris of the barn owl. The principle of the time coding to space coding converter implemented in this nucleus is sketched in the left panel. Synchronous inputs from the right and left ear lead to the discharge of the neurone in the middle of the row. The greater the interaural delay, the farther from the centre of the row the discharging neurone, for which the input interaural aural delay is exactly compensated by the differential conduction delays in the transmitting fibers. The panel on the right illustrates a possible mechanism for achieving a very fine coincidence discrimination, despite the anatomically predicted spreading of the signal in the terminal axonal arborisations in the nucleus laminaris afferents. It is assumed that a subset of synapses are selected according to their common phase by a Hebbian mechanism of plasticity. (Modified from Gerstner et al, 1996). 
ficial layers of the visual cortex, furnish another example of neuronal clocks (Gray \& McCormick, 1996). When stimulated, chattering cells emit a series of bursts at an extremely high intraburst frequency (up to $800 \mathrm{~Hz}$ ), and a variable interburst frequency depending on the level of excitation of the cell (Fig. 3). It has been speculated that the rapid firing within a burst could be useful for achieving a postsynaptic temporal summation of great efficacy, and that the interburst frequency may constitute a major source of evoked $\gamma$-band oscillations in the local populations of cortical neurones. It should be noted that the frequency of discharge of a chattering cell is not fixed, but rather depends on the controllable level of depolarization of its membrane, as experiments using intracortical injections of currents seem to show (see Fig. 3, right panels).

It is nowadays usual to oppose two modes of functioning of neurones, namely the integrateand-fire mode, and the coincidence-detector mode. Detection of interaural delays in owls is a good example of extreme specialization of neurones as sharp coincidence detectors. The concept of coincidence detector, however, may well be enlarged to that of phase detector (PD). Instead of giving a binary ("yes" or "no") response, according to whether a coincidence has (or has not) been detected, a phase detector gives a gradual, analogue response to small time delays between two inputs (or two groups of inputs).
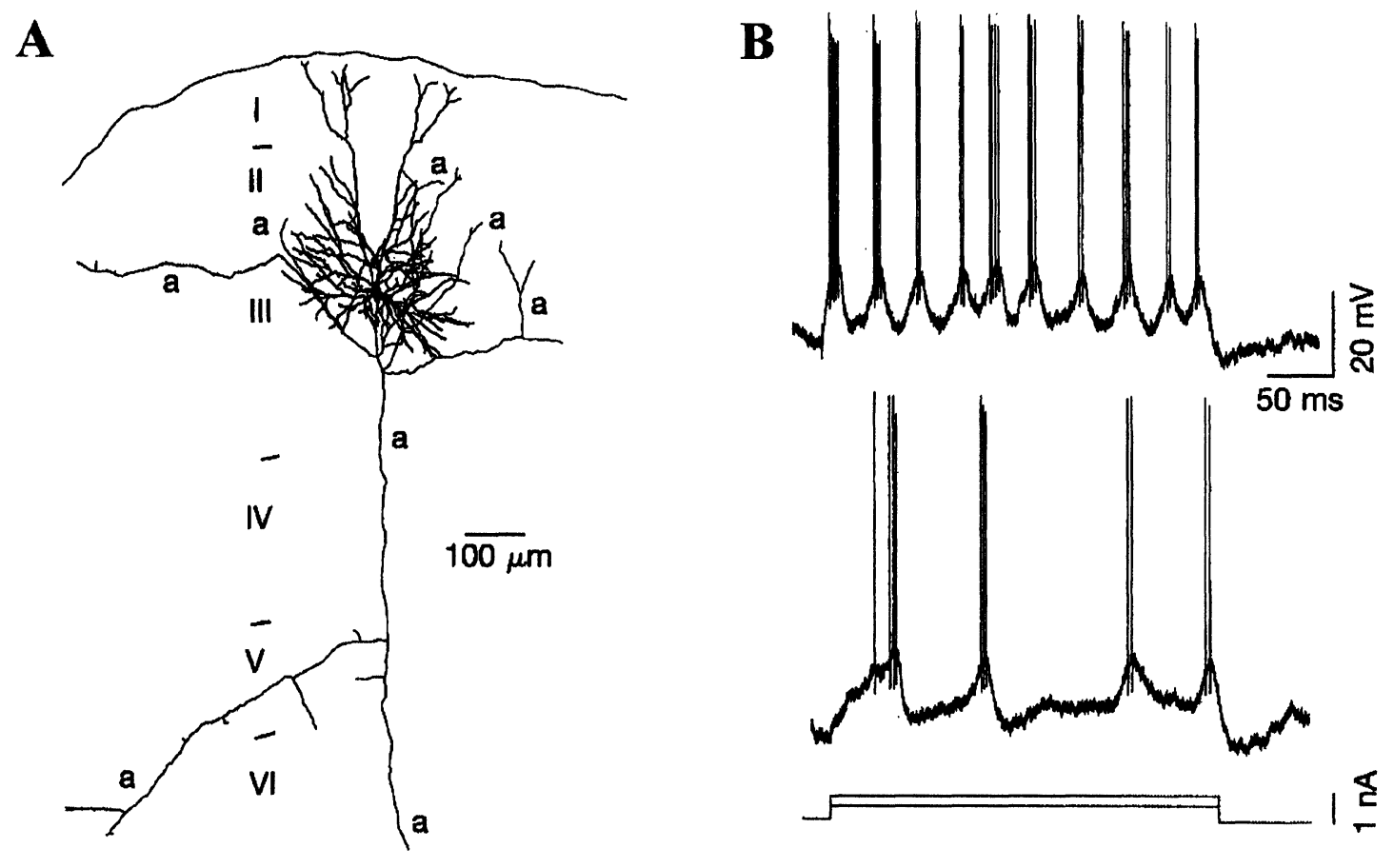

Fig. 3: (A) Camera lucida reconstruction of a cell characterized as a chattering cell, having a spiny pyramidal morphology, and its soma located in layer III of the visual cortex of a cat. The axon branches and sends out collaterals in layers II, III, and V. Local axon collaterals are indicated by the letter ' $a$ '. The axon also projects into the white matter (not shown). (B) Responses of the cell to two different levels of intracellular depolarizing pulses. In both traces, the cell exhibits high-frequency bursts of action potentials that are characteristics of chattering cells. The pattern of burst firing is rhythmic when the cell is depolarized strongly. At lower levels of depolarization, the cell continues to burst, but in a temporally disorganized manner. There is no apparent evidence of an underlying subthreshold membrane oscillation in the lower trace. (From Gray and McCormick, 1996.) Reprinted with permission. (C1996 American Association for the Advancement of Science. 
Thus a PD is an appropriate device to compare an external signal (a time interval between two excitations) to an internal signal provided by an internal oscillator.

A phase detector coupled with a controllable internal oscillator makes up what is called in the literature a phase-locked loop (PLL). The possible embodiment of neuronal PLLs in the central nervous system was proposed in particular by Ahissar \& colleagues (Ahissar \& Vaadia, 1990; Ahissar et al., 1997; Ahissar, 1998). These authors suggested that PLLs could, in fact, be
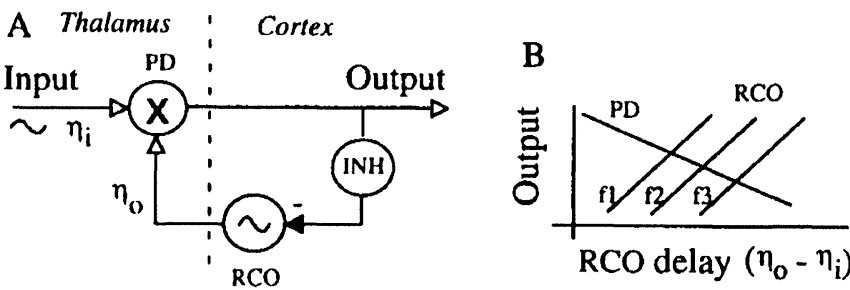

C ISI Histograms

D Individual ISIs in single trials
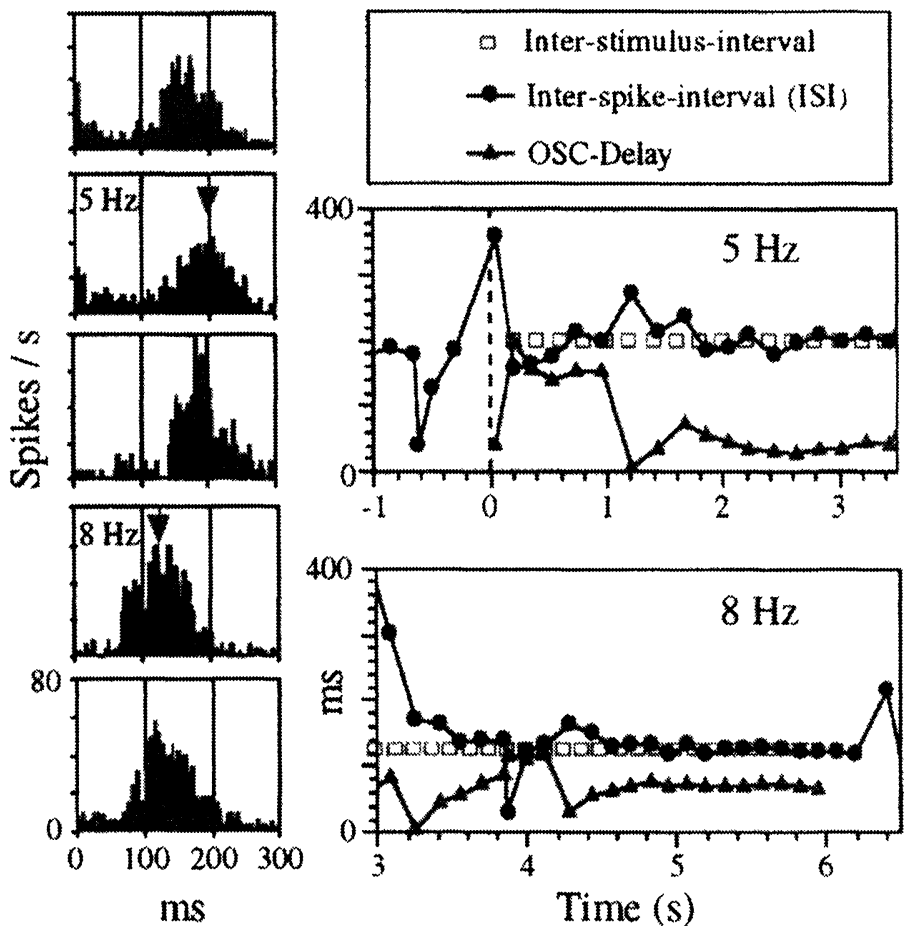

Fig. 4: (A) Principles of an inhibitory Phase-Locked Loop (PLL) decoder. INH, inhibitory neurone(s); RCO, inhibitory connection; $\eta_{0}$, time of the recent RCO spike; $\eta_{i}$, time of the recent input spike; PD, phase detector. B: PLL transfer functions. The transfer functions should be monotonic, but not necessarily linear, within the PLL's working ranges. The PD's output decreases as the RCO delay $\left(\eta_{0}-\eta_{i}\right)$ increases (PD curve). The RCO's firing time is delayed as the PD's output is increased (RCO curves). The exact relation between the two transfer functions depends on the input frequency ( $\mathrm{f} 1>\mathrm{f} 2>\mathrm{f} 3$ ); stable (crossing) working points for higher frequencies are associated with larger RCO delays and lower outputs. (C, D): Frequency locking of a single-cell cortical oscillator recorded from layers 2-3 of the barrel cortex of an anaesthetized rat during the stimulation of whisker E2 with square-wave stimuli. (C): Interspike intervals (ISI) computed during the entire stimulation periods (black) and the interleaved spontaneous periods (gray). Arrows point to the interstimulus intervals. (D): Lock-in dynamics during single stimulus trains. ISIs, inter-stimulus intervals and OSC-delays (triangles) are plotted as a function of time. Time 0 and the dashed line denote the beginning of a stimulus train. Note the 1:1 firing (one OSC spike per stimulus cycle) and constant phase difference during stabilized states. In the trial presented at bottom, the OSC remained "locked" for two additional cycles after the stimulus train ended. (From Ahissar et al, 1997). Reprinted with permission. (C)1997 National Academy of Sciences, U.S.A. 
numerous in various parts of the sensory system, either at the thalamic or at the cortical level. Indeed, an investigation of the somatosensory cortex of rats and guinea pigs provided evidence for the abundant presence of cells with oscillatory-type discharges. The oscillations were categorized according to their frequency ranges, and it was found that, in these preparations, oscillators could be grouped into three categories according to their frequency range:

1. around $1 \mathrm{~Hz}$ (these oscillators are perhaps driven by the anesthesia or by respiratory movements),

2. $10 \mathrm{~Hz}$ (matching rather well the usual behaviorally observed whisking frequency), and

3. $100 \mathrm{~Hz}$.

The local origin of the oscillations and the controllable nature of the oscillators in the $10 \mathrm{~Hz}$ band were tested by applying glutamate through glass pipettes that surround the recording electrode. It was observed that in most oscillators, the frequency consistently increased after glutamate injection. Whether such oscillators were involved in PLL circuits, in relation to the processing of somatosensory information that is provided by the whisker's motion, was then investigated.

Let us examine the functioning of a PLL (Fig. 4, panels A and B). As we just stated, the PD does not give a binary response, but rather a graded response according to the time difference between the external input $\left(\eta_{i}\right)$ and the input from the ratecontrolled oscillator (RCO) $\left(\eta_{0}\right)$. For an oscillatory input, the output of the PD is thus a decreasing function of the phase lead of the input $\left(\eta_{i}-\eta_{0}\right)$. As a consequence of the negative feedback, for a given frequency of the input $f_{1}$, the frequency of the RCO is an increasing function of the phase lead. There will be an equilibrium point, at which both the input frequency and the RCO frequency equalize, with a fixed phase lag of the RCO. Simulations show that the greater the difference between the rest frequency of the RCO and the frequency of the input, the greater the phase lag.

Figure 4 (C and D) displays the phenomenon of frequency locking, which is observed at a single cortical oscillator (OSC) recorded from layer 2-3 of the barrel cortex of an anaesthetized rat ("proper" frequency $\sim 6 \mathrm{~Hz}$ ) during stimulation of whisker E2 with square-wave stimuli. Two examples of the dynamics of the system are shown in panels $\mathrm{C}$ and $\mathrm{D}$. Interspike intervals (ISI) are shown as black dots, interstimulus intervals as open squares, and OSC delays as filled triangles. Time 0 and the dashed line denote the beginning of a stimulus train. Note the 1:1 firing (one OSC spike per stimulus cycle) and the constant phase difference after stabilization. This phase difference is greater at $8 \mathrm{~Hz}$ than at $5 \mathrm{~Hz}$, in agreement with the rule that the farther the applied stimulation from the proper frequency, the greater the phase difference.

The somatosensory system is obviously not the only one to contain oscillatory clocks. In fact, every neocortical area seems to contain such neurones. We have already mentioned the case of chattering cells, observed in the primary visual cortex (Gray \& McCormick, 1996). These superficial layer neurones are ordinarily active when, and only when, there is a sufficient level of cortical activation from lateral inputs or from the modulatory afferents coming, for instance, from the brain stem. The frequency of the chattering cells increases with this level of cortical activation. Chattering cells might also be involved in PLL circuits, although the existence of such circuits is unclear at the moment.

Let us return for a moment to Fig. 1. Laurent and his collaborators observed that:

a) the oscillations were brief (50-250 ms),

b) during a limited time, spikes in different principal cells of the antennal lobe tended to synchronize closely within the oscillation cycle, although they desynchronized at later periods of the oscillation,

c) the dynamic sequence of AL cells, which are excited (or inhibited) during the successive periods of the oscillation, is related to the nature of the stimulus.

These observations confirm that in this case, one function of the oscillations is to provide a tempo, but at the same time suggest a limitation of the concept of oscillations as a mechanism to provide precise synchronization because this 
synchronization seems to be progressively lost as we proceed over the succession of periods (Laurent et al., 1996). That synchronization and oscillations should be dissociated is also suggested by the observations made on the visual cortical areas of mammals showing that oscillations of local populations of neurones seems to follow, not to precede, synchronization.

\section{OSCILLATIONS AS A SPECIAL CASE OF PRODUCING RPEATING PATTERNS}

Synchronization implies that excitation impinging on a given neurone through several afferents should arrive in a narrow window of time. We have already seen that in the case of coincidence detector cells of the nucleus laminaris of the owl, excitation from afferent spikes coming from the nucleus magnocellularis spreads over the terminal axo-dendritic arborization over a span of time of about $250 \mu \mathrm{s}$. Verifying to what extent time constraints in the spreading of inputs on a single neurone apply also in other neurones in the CNS is important, for instance in the visual system, in which a looser time tuning might be of less consequence than in a system devoted to interaural delay detection.

Figure 5 displays the camera lucida reconstruction of a magnocellular thalamocortical neurone of a monkey, as Freund and colleagues published 10 years ago (Freund et al., 1989). In

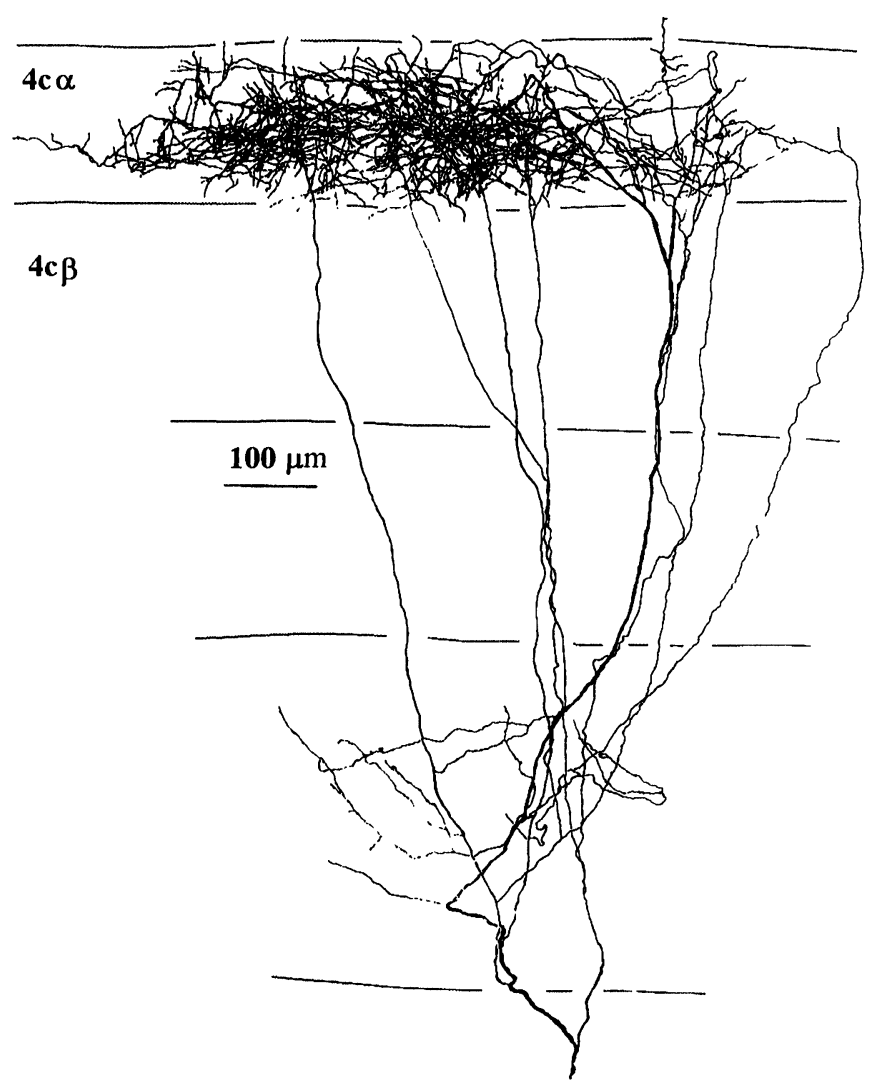

Fig. 5: Camera lucida reconstruction of a magnocellular axon in the monkey striate cortex. It had an off-center receptive field. The center size was $0.5^{\circ}$ in diameter at a position of azimuth $7.5^{\circ}$, elevation $-3.0^{\circ}$. The axon was driven by the ipsilateral eye and responded well to low-contrast, broad-band stimuli. The terminal arbor is restricted to the upper part of layer 4C; only a few collaterals terminate in layer 6. A top view (from the pia) shows that the boutons form three distinct, unevenly populated, clumps (not shown). Scale: $100 \mu \mathrm{m}$. (From Freund et al., 1989). (C1989 J. Comp. Neurol. Reprinted by permission of Wiley-Liss, Inc., a division of John Wiley \& Sons, Inc. 
this example, the thalamocortical neurones, which were studded with large en passant boutons, branch even before leaving the white matter, although the bulk of wide terminal arborization develops only in the layer $4 \mathrm{C} \alpha$. This neurone gave about 3,200 boutons in three different cortical clumps (hardly visible in this side view of the neuronal arborization), with a total elongation of the terminal arborization of about $1.8 \mathrm{~mm}(0.8$ $\mathrm{mm}$ from clump centre to clump centre). Each bouton had roughly $2 \pm 1$ synapses. Each cortical target neurone receives $8-10$ boutons, therefore the number of target cells of a single thalamocortical neurone is of the order of 400 . The interval between successive boutons is typically about 15 microns, which means that each is reached after an interbouton delay of $5 \mu \mathrm{s}$ (assuming a value of $0.3 \mathrm{~m} / \mathrm{s}$ for the conduction velocity, see below).

The same typical architecture is visible for parvocellular thalamocortical cells as well (not shown), except that in this case, the terminal arborization is clearly concentrated in layer $4 \mathrm{C} \beta$, and the total spread of the arborization is even narrower: only about $400 \mu$.

What about the time dimension of the spreading of excitation on a given target cell and along the chain of targeted cells? Light micrography does not reveal much about the dynamics of the transmission of a spike through the terminal arborization of an axon. A detailed simulation (of transcallosal fibers) was recently performed by Jean-Christophe Houzel and colleagues in Lausanne (Houzel et al., 1994; Innocenti et al., 1994).

Figure 6A presents a transcallosal fiber, connecting two cells belonging to the boundary region between areas 17 and 18 of the visual cortex of the right and left hemispheres. This axon presents a simple branching, and the terminal arborization innervates two clusters in two cortical columns. Colors code for the time of activation in microseconds. It is remarkable that all boutons located in a given cluster are activated within a very small window of time, again of the order of $250 \mu \mathrm{s}$, and that the synchrony of activation between the two clouds is almost as precise as the synchrony inside of a given cloud (not more than $400 \mu \mathrm{s}$ ) (see histograms of times of activation for all boutons and each cloud separately). Times are counted from the time of passage of a spike in the medial line of the corpus callosum.

In one other example (Fig. 6B), the case of a more complex architecture is examined. Here the terminal arborization innervates up to five distinct clusters in the contralateral area 17-18. In addition, the two main branches of the axon (colored in blue and in yellow in the middle panel of the figure) reconverge on the same synaptic cluster. Time-spreading expectations in this case were as follows: in each cluster receiving only one main axonal branch, the maximum time spread was $<200 \mu$ s. The time spreading in the cluster receiving inputs from each of the two main branches was even sharper, about $100 \mu \mathrm{s}$. The maximum average time delay between the five clusters was $300 \mu \mathrm{s}$. Although these figures are subject to some uncertainty, depending upon the exact value of conduction velocity in the thinner branches of axonic arborization (here the validity of the Rushton-Waxman linear law between velocity and diameter had been assumed), the submillisecond spreading in activation times within and across synaptic clusters of a given axonic arborization should be considered as a robust result.

\section{MODELING SYNCHRONOUS ACTIONS IN THE CNS}

How should we incorporate these precise temporal requirements into our models of information processing in the brain? Let us compare here two models that were proposed more than a decade ago: The Abeles synfire chain on the one hand, and the Strehler-Lestienne hebbian chain on the other (Fig. 7).

In the synfire chain model, each neurone is richly connected in a diverging-converging mode, and the neurones fire when a sufficient number of inputs are received synchronously. Modeling this type of chain has proved that excitation can propagate successfully through the various steps. The synchronization of neuronal inputs and outputs at 
each step is very rapidly achieved (after a few initial steps) and is maintained throughout the chain (Abeles, 1989). Picking at random a few cells belonging to a synfire chain should lead to the observation of the characteristic patterns of interspike intervals across neurones. Some evidence has been published for the occurrence of very precise patterns of this sort, with a precision of pattern reproduction of the order of one $\mathrm{ms}$, when interspike time intervals may be as large as
A
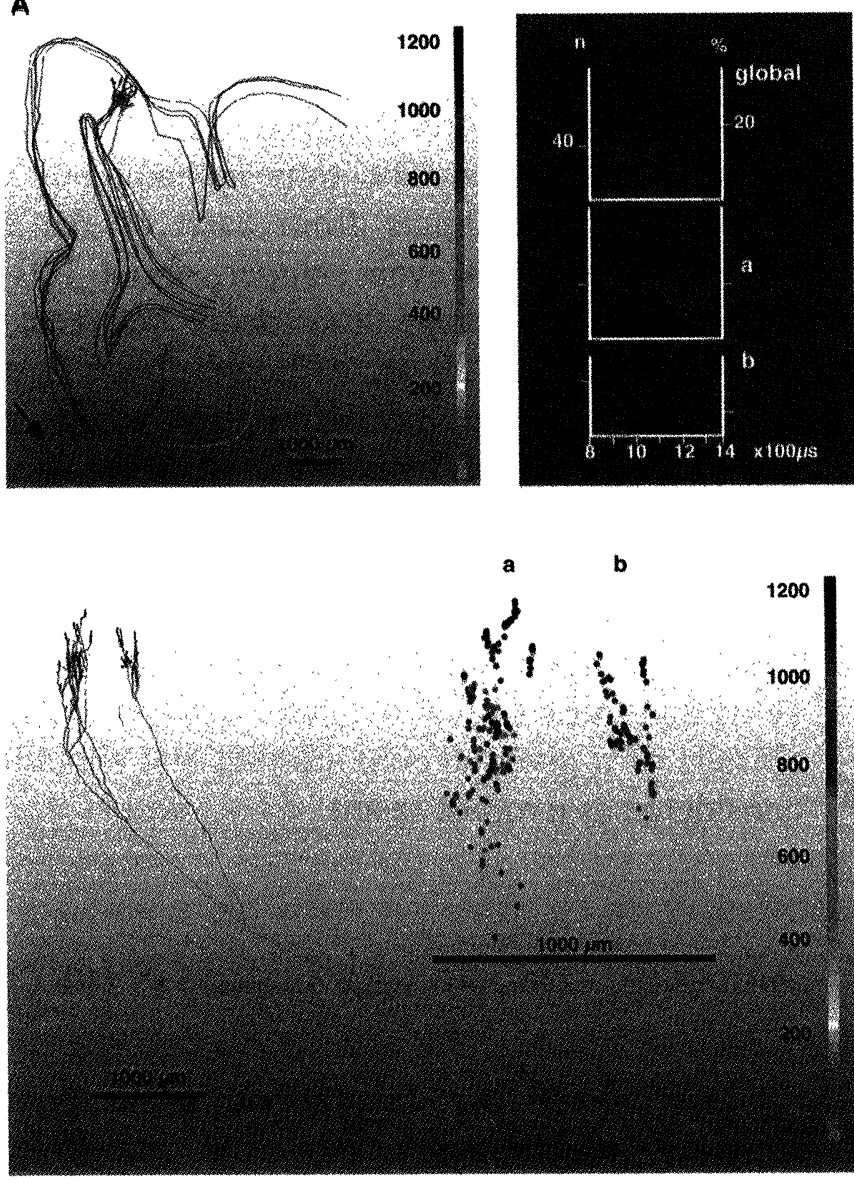
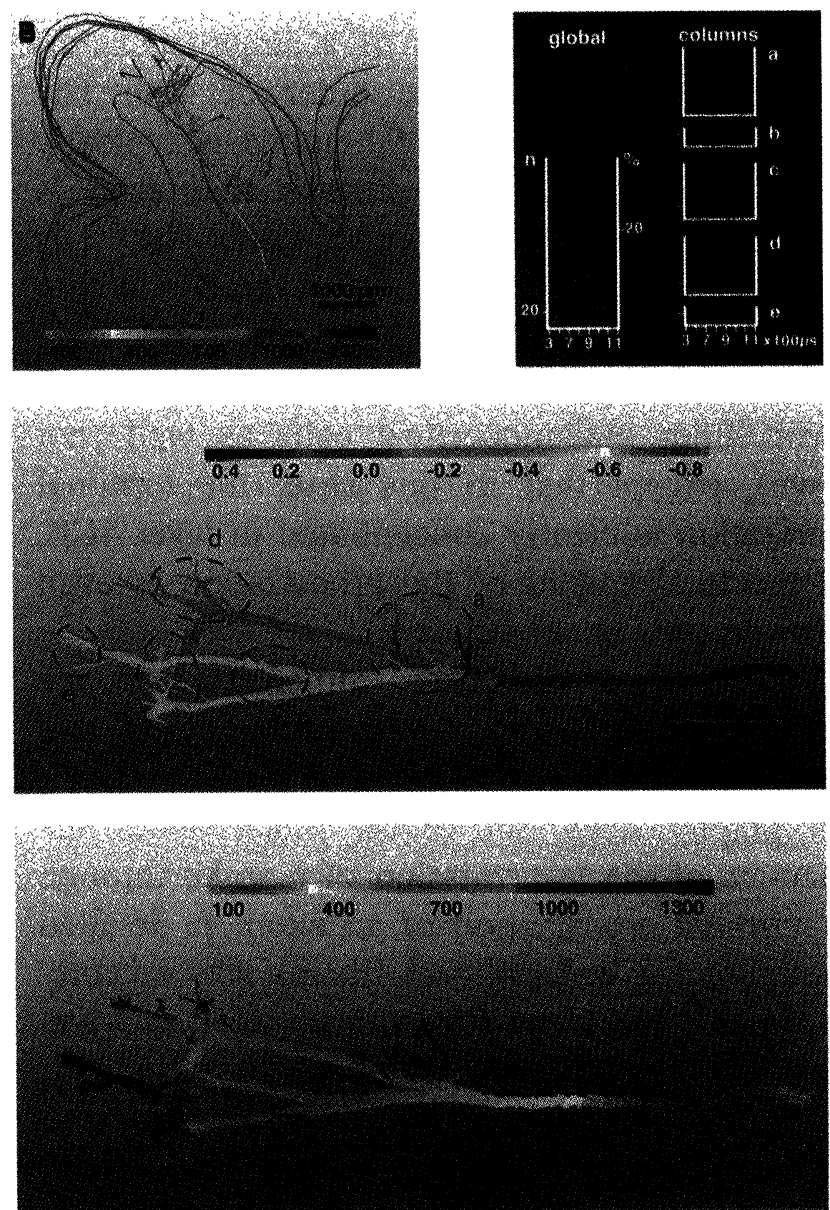

Fig. 6: Two transcallosal fibers, originating near the border between areas 17 and 18 of the visual cortex of a cat and innervating the contraletral homologous area, were anterogradely labeled with biocytin, reconstructed in 3-D from serial sections. Their architecture was fed into a simulation software (Maxsim; see Tettoni et al, 1993) for allowing to study the temporal activation profile of their terminal arborization. The time of activation, counted from the crossing of the interhemispheric boundary, is coded in false colors, according to scales shown (A) This transcallosal fiber has an early split, long before the terminal arborization. The two main branches innervate two distinct clusters located in two distinct microcolumns, about $600 \mu \mathrm{m}$ apart. All terminals boutons, within or across the two clusters, receive an action potential within a window of time about $400 \mu$ s wide. (B) This more complex terminal arborization innervates five distinct clusters, labeled a,b,c,d,e. One branch (in yellow in the topographical map, middle panel) innervates clusters a, b, c, and $\mathrm{e}$; the other branch innervates clusters a and $\mathrm{d}$ and then, by a reconvergence, cluster $\mathrm{e}$. All terminal boutons within a cluster receive the action potential within $200 \mu \mathrm{s}$ or less, and the spread across the clusters does not exceed 300 $\mu \mathrm{s}$. (After Houzel et al., 1994 and Innocenti et al., 1994). 
several hundreds of ms. This observation may require qualification, however, because it has been shown quite recently (Richmond, this issue) that the large variability of most cortical discharges usually does lead to an underestimation

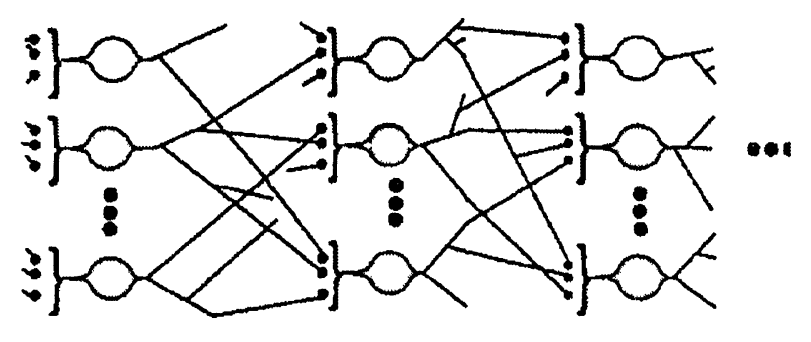

Synfire chain (Abeles)

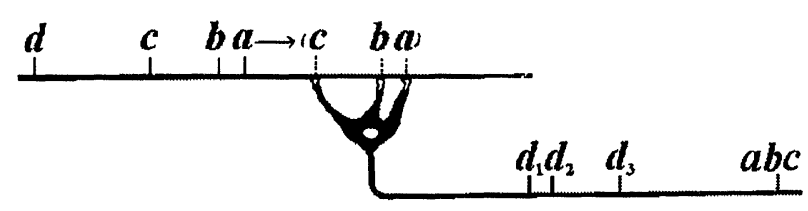

Hebbian chain (Strehler-Lestienne)

Fig. 7: Two models of synchronization. The synfire model developed by $M$. Abeles (1989) considers that synchronization is the result of both the functioning mode of neurones (as coincidence detectors, rather than integrate-and-fire devices) and the partial diverging/converging chain structure of the connectivity. Modeling predicts that synchronization at the ms or subms level is achieved after a few steps in the chain, and is robust all along the chain. The model predicts also the detection of precisely repeated patterns in the firing times across neurones. In the early form of the StrehlerLestienne (1987) model, synchronization across different clusters of synapses is achieved in a single neurone, by the precise tuning of the successive intervals in the spike train. Fast synaptic plasticity, due to the repeated input of the tuned patterns, allows a faithful transmission of the tuned intervals (except for time order inversions). The narrow synchronization of inputs in single cells makes this model unrealistic, but it may be extended to chains of neurones. (Modified from M. Abeles, 1989 and from Lestienne and Strehler, 1987.) of the probability that such recurring patterns may arise by chance

The Strehler-Lestienne model is of a different nature. Initially, it was imagined that, thanks to the geometry of the axo-dendritic arborizations and the variable conduction delays that this geometry introduces, interspike intervals could be used to achieve a very precise synchronization of inputs in a single target cell. By a Hebbian mechanism of plasticity, repeated bombardment with an appropriate pattern of spikes would increase the efficiency of the concerned synapses, making the line in a way specialized for the identification of this or a limited set of patterns carried along an axon and distributed over the previously sensitized cluster of synapses would and allowing a faithful downstream transmission of them for a short while. Thus, a single pulse generate a (time-inverted) image of the original sequence of pulses, which is responsible for the discharge of the target cell in the first place (Lestienne \& Strehler, 1987). This model is obviously unrealistic if applied to single target neurones because, as we stated, the spread of time of arrivals at the different synapses on a single target cell is only about $250 \mu \mathrm{s}$, a value much shorter than that of the refractory period; in other words, two successive spikes, by necessity separated by a few ms or more, cannot contribute to the synchronization of EPSPs in a single target cell. The model might apply to a chain of neurones, however, and it has two appealing characteristics:

1. the suggestion of a submillisecond precision in spike timing, and

2. the possibility of rapid switching over lines in a chain of neurones, thanks to a postulated phenomenon of fast synaptic plasticity (Markram et al., 1997).

The first characteristic is supported by anatomical observations and modeling as well. In various single cell spike trains (Strehler \& Lestienne, 1986; Lestienne \& Tuckwell, 1998) in LGN and visual cortical discharges of awake monkeys (Oram et al., 1999), spike patterns (in particular, doublets and triplets of spikes) have been shown to repeat themselves with a ms or sub-ms precision in rather narrow windows of time. 

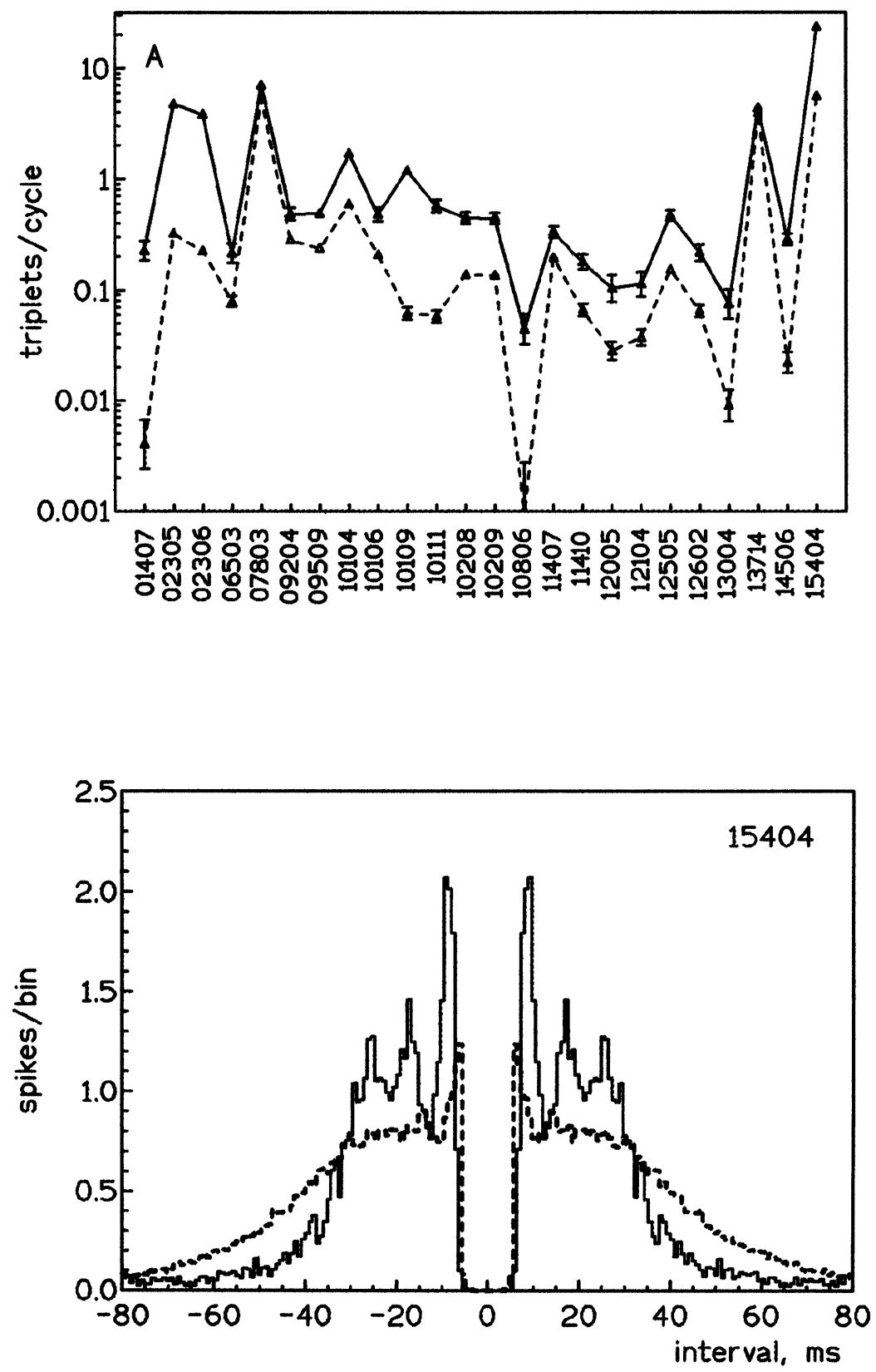

Fig. 8: (A) Detecting replicating triplets in the mitral cell discharges of freely breathing rats. In this collection of 24 records (record labels in abscissa) chosen for their significance (out of 118 records), the rate of production of precisely replicating triplets of spikes (full triangles and full lines) is compared with that in Non Homogeneous Poisson Process (NHPP)-generated spike trains, having the same PSTHs and similar refractory periods (open triangles and broken lines). The logarithmic scale emphases the logarithmic parallelism in the trends of production rates in the two sets of spike trains, and the fact that in physiological data, replicating patterns can be as high as ten times more numerous than in simulations. In the remaining records, where the production of replicating patterns is smaller, there is still a consistent excess of replicating patterns in the physiological data, as compared to simulations. (B) The limited autocorelogram (up to the third other spike in the train) for this record displays the strong temporal organization of the discharge, which is absent in the corresponding NHPP simulation. Such a temporal organization, although reminiscent of oscillations, may in fact reflect the high level of production of repeating patterns of spikes. (From Lestienne et al., 1999; (O1999 Europ. J. Neurosci.) 
Note that to be used by the brain, such replicating patterns do not necessarily have to be produced by specific, stimulus related processes. As a matter of fact, the production of replicating patterns is primarily related to the firing rate. The higher the firing rate, the higher the probability to get in a given time window several patterns that fulfil the replication requirements. In fact, the number of replicating triplets detected in the experiment mentioned above was a highly nonlinear function of the number of spikes in the trial (see B.J. Richmond et al, this issue, Fig. 5). This non-linearity, already predicted for the Poisson process ten years ago (Abeles \& Gerstein, 1988), raises the possibility that their production might be correlated to the large variability that has been observed in the statistics of spikes per trial. In episodes of high frequency, generated by large fluctuations in the variability, the number of replicating patterns occurring by chance is much higher than predicted, even by a time-varying (non-homogeneous) Poisson process. But once again, such a semi-stochastic model of the production of precisely replicating patterns would not imply that they are not important or meaningful for information processing. It would mean only that spike counts or repeating pattern counts are two equally possible ways to assess the meaning of neuronal discharges (the true relevance of either way resting ultimately on the physiological decoding system used by the brain).

Another situation in which an abundant production of precisely replicating triplets has been found is the analysis of mitral cell discharges of anaesthetized rats (Lestienne et al., 1999) (Fig. 8, panel A). In this study, rats were submitted to an odor excitation, and the number of replicating triplets in mitral cell responses was compared with the result of simulations using a non-homogeneous process (NHPP), exactly reproducing the time course of the discharges along each respiratory cycle. The number of replicating triplets was the number of times that two identical triplets were found (with a tolerance of $0.5 \mathrm{~ms}$ for each time interval) in a sliding window of time of $200 \mathrm{~ms}$ duration, each interval in a triplet being limited to values $\leq 50 \mathrm{~ms}$. The authors showed that in a large fraction of the records under odor stimulation, precisely replicating triplets were largely in excess of the predicted rate of production on the basis of NHPP simulations, this difference being at times as large as a factor of ten.

A detailed analysis of one of the most striking productions of replicating patterns is displayed in Fig. 8B. The left histogram shows the autocorrelogram of limited range (plotting the correlation between the emission of a spike and the emission of other spikes only up to the third spike before or after it), as observed in the real data (continuous line) and compared with that observed in NHPP-generated simulations (dashed line). A striking structure appears, which is reminiscent of oscillations but is more evident in inspection of such limited range correlograms than in the usual, all-order autocorrelograms. This observation confirms the highly transient nature of the phenomenon observed. In this case, one should also note that the variability of the response in terms of spikes per trial is not larger but rather narrower than its expectation from the NHPP model, in contrast to observations in other parts of the CNS, including the LGN discharges of awake monkeys, but similar to observations in ganglion cell discharges (Berry et al., 1997). This difference makes a purely stochastic production of replicating patterns that is unlikely in the case of olfactory stimulation of mitral cells.

It should also be noted that an analysis of the same LGN and mitral cell data, using the traditional tools (autocorrelograms and power spectra) for the detection of oscillations patterns, was much less successful than that for the detection of replicating patterns, suggesting that pattern replication is perhaps a much more robust or more prevalent phenomenon than oscillations. The latter would then be considered a special case of the former (when replicating intervals have the same value and replication is renewed over a relatively large number of periods).

Finally, what are the possible decoding mechanisms of such replicating patterns? We have seen that in the case of thalamocortical cells, the average distance between two target cells in the cortex from a single thalamic relay cell $(800$ $\mu)$ and the probable value of conduction velocity 
in the smaller axonal fibers $(0.3 \mathrm{~m} / \mathrm{s})$ do not leave much hope for a Strehler-Lestienne type of detection mechanism between a single pair of connected neurones. The maximum time interval for the synchronization of inputs in such a configuration is less than $3 \mathrm{~ms}$ (unless some delay mechanism other than conduction is taking place). A possible candidate for a mechanism of this kind is the interposition of inhibitory interneurones, as seen for instance in the functioning of the sonar of the bat (Saitoh \& Suga, 1995). Some results exist, however, that tend to indicate that the fine temporal organization of spike trains might indeed have a real significance for information processing in the brain at the multi-synaptic level. One such experiment consisted of recording
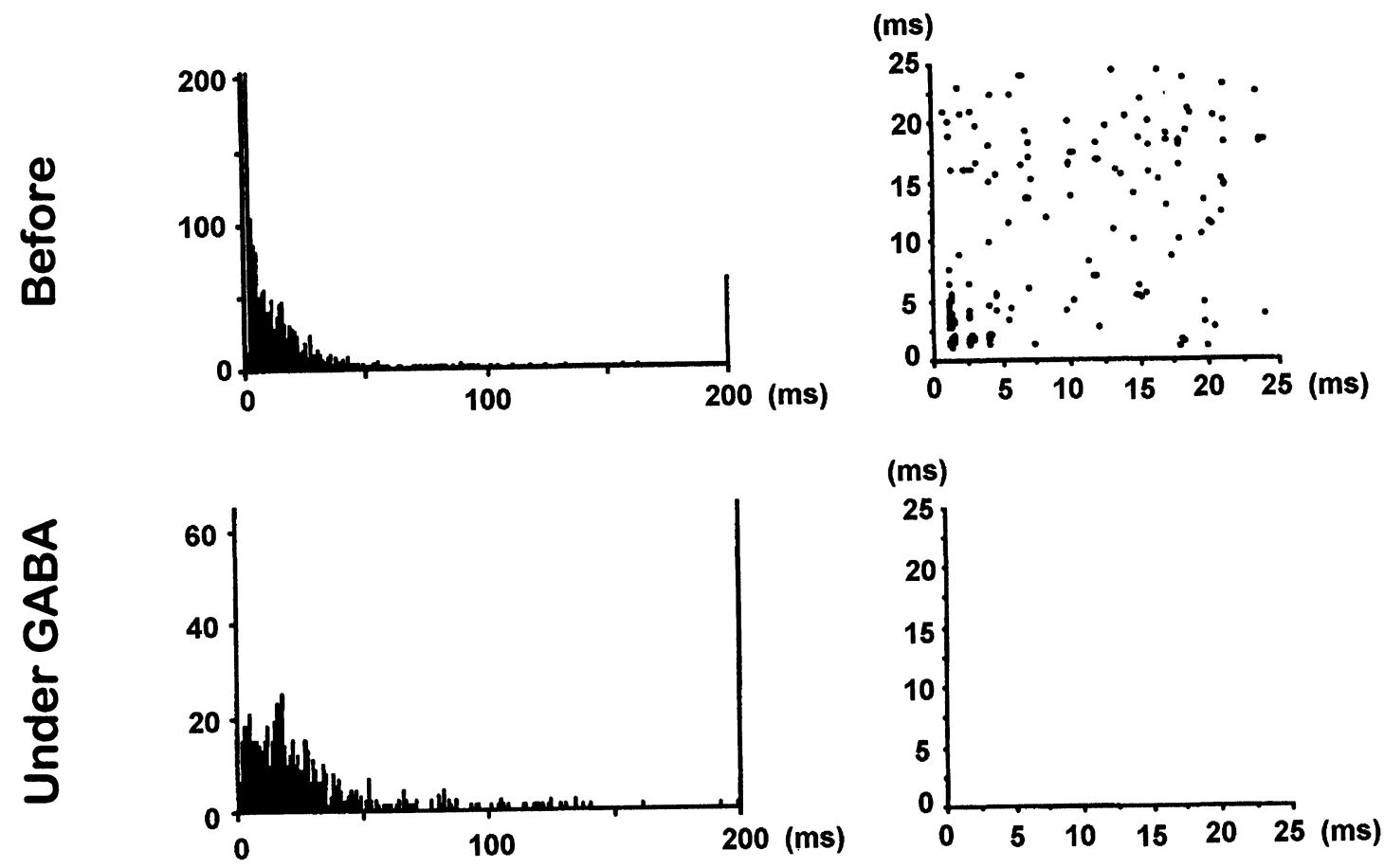

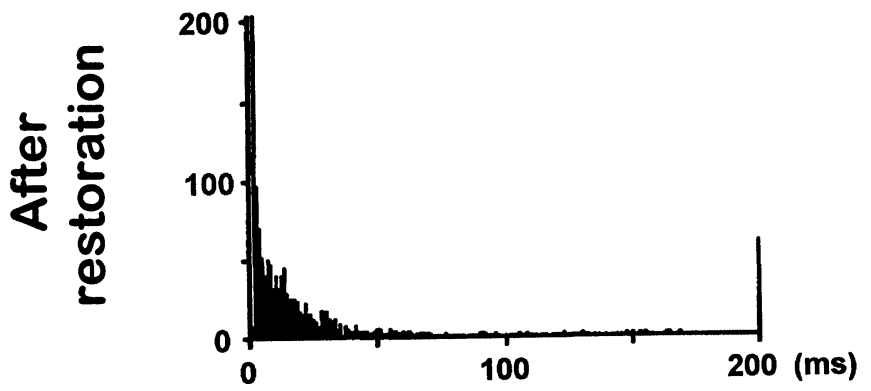

TIH

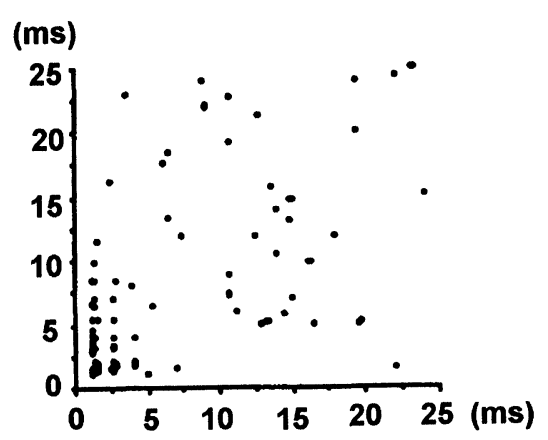

$(a, b)$ plot

Fig. 9: Precisely replicating triplets detected in the LGN spike trains of an anaesthetized cat are disrupted during a cortical application of GABA. Temporal structure representations of replicating triplets detected in an impulse train before, during, and after a local cortical application of GABA. The record has been kept on 20 runs of $2.5 \mathrm{~s}$ duration each. After the injection of GABA, replicating triplets were no longer detected in the spike train. The bottom two-dimensional plot indicates an almost complete recovery of the triplet production after dissipation of GABA. The Time Interval Histogram (TIH) was only slightly and reversibly affected during GABA application. (From Beaux et al., 1992). 
simultaneously from two single cells, one in the LGN and the other in the primary visual cortex in an anaesthetized cat, submitted to visual stimulation in form of drifting gratings. Both cells had overlapping receptive fields, increasing the chance that they be connected by corticothalamic fibers. Figure 9 shows that when GABA was iontophoretically applied to the region of the cortical cell, thus silencing the corticothalamic pathway, the number of the replicating patterns found in the thalamic discharges was greatly reduced and their temporal structure was considerably modified, and sometimes even completely abolished, although the rate of discharge of the LGN cells was not modified by the GABA injections (Beaux et al, 1992). Similar results have since been published (Funke et al., 1996; Wörgotter et al., 1998). These reports encourage further verification in awake, performing primates.

\section{DISCUSSION AND CONCLUSION}

The exquisite architecture of connections in the brain, the matching of neuronal process diameters and the subtlety of synaptic layout in glomeruli or spines are powerful indications that synchronization and temporal organization are key concepts in the functioning of the CNS, even though very little is understood about their mechanisms. The survey of the functioning of several sensory systems that we have made here strongly supports this view, at variance with the claim still firmly held by some researchers that nothing that we know of the CNS suggests any coding but the firing rate (Shadlen \& Newsome, 1998). On the contrary, it seems to me that we have now several clear indications that an organism uses in different parts of its sensory systems, both time-to-place and place-to-time encoders and decoders. For instance, in the protocerebrum of insects and in the auditory system of owls, time-to-place conversions of odors or sounds take place, whereas in the somatosensory system of the rats and guinea-pigs, rather a placeto-rate conversion takes place.
If, therefore, timing and synchronization play an important role in the CNS, then a better understanding of the mechanisms involved is very important. Oscillation is one of the most commonly evoked mechanisms, although the definition of oscillation in a neuronal system lacks the precision that it has gained in physics and does not seem to meet all the precision timing requirements that seem to be generally fulfilled by the brain. Indeed, on several occasions, we have found indications suggesting a millisecond or even a submillisecond temporal organization of spike discharges. Sometimes such situations are claimed to belong only to rare, highly specialized systems, in situations of "hyperacuity", but the modeling of a simple transcallosal fiber shows that stringent timing conditions seems to be a rather common situation in the brain.

In the recent past, there has been a tendency to fuse (or to confuse) the two concepts of oscillation and synchronization into one, speaking of "coherent oscillation" or "synchronous oscillations", especially in reference to the binding problem, as if the congruence of the two notions were granted. Let us take once again the example of the olfactory discharges in the antennal lobe of a locust. We now know that the important point is to achieve synchronization of the inputs at level of the calyx of the mushroom body. These neurones, called the Kenyon neurones, have been shown to act essentially as coincidence detectors, in the sense that they nonlinearly integrate the inputs impinging on them (Laurent G., personal communication).

Oscillations per se cannot fulfil the function of synchronizing inputs that may be several tens of milliseconds apart. On the other hand, inhibitory interneurones seem to have the capacity to introduce the appropriate time delays between neurones. A network of inhibitory feedback neurones, such as that which has been shown to exist in the mushroom body (Bicker et al., 1985), could well fulfill the function of decoding time delays. The temporal structure induced by these synchronizing devices might resemble oscillations if repeats of simple patterns are needed, without really being oscillations. On the 
other hand, more classic prolonged oscillations may well apply to other situations and have other functions, either in attentional mechanisms, as was suggested in particular by Rougeul-Buser (1994), or in the process of memorizing items, as the oscillatory nature of hippocampal discharges may indicate (Buzsaki \& Chrobak, 1995; Traub et al., 1996). Exploration of the precise, although not necessarily deterministic, (Richmond, this issue) mechanisms sculpturing the discharges is still widely open until we fully understand their function.

\section{REFERENCES}

Abeles M, Gerstein G. Detecting spatiotemporal firing patterns among simultaneously recorded single neurons. J Neurophysiol 1988; 60: 909-924.

Abeles M . Neural codes for higher brain functions. In: Markowitsch HJ, ed, Information Processing in the Brain. Views and Hypotheses from a PhysiologicalCognitive Perspective, Toronto: Hans Huber 1989; 225-237.

Ahissar E, Haidarliu S, Zacksenhouse M. Decoding temporally encoding sensory input by cortical oscillations and thalamic phase comparators. Proc Natl Acad Sci USA 1997; 94: 11633-11638.

Ahissar E. Temporal-Code to Rate-Code conversion by neuronal phase-locked loops. Neural Computation 1998; 10: 597-650.

Ahissar E, Vaadia E. Oscillatory activity of single units in a somatosensory cortex of an awake monkey and their possible role in texture analysis. Proc Natl Acad Sci USA 1990; 87: 8935-8939.

Beaux J-C, Lestienne R, Imbert M, Grandjean B Cortical modulations of fine temporal structures of impulse trains in the dorsal lateral geniculate nucleus of the cat. CR Acad Sci Paris III 1992; 314: 31-36.

Berry MJ, Warland DK, Meister M. The structure and precision of retinal spike trains. Proc Natl Acad Sci USA 1997; 94: 5411-5416.

Bicker G, Schäfer S, Kingan TG. Mushroom body feedback interneurones in the honeybee show GABA-like immunoreactivity. Brain Res 1985; 360: 394-397.

Briten KH, Shadlen MN, Newsome WT, Movshon JA. The analysis of visual motion: a comparison of neuronal and psychophysical performance. J Neurosci 1992; 12: 4745-4765.

Buzsaki G, Chrobak JJ Temporal structure in spatially organized neuronal ensembles: a role for interneuronal networks. Curr Opin Neurobiol 1995; 5: 504510.
Delage Y. Le rêve. Etude psychologique, philosophique et littéraire. Paris: Presses Universitạires de France, 1919. [See Herculano S. Yves Delage: Neuronal Assemblies, Synchronous Oscillations, and Hebbian Learning in 1919, The Neuroscientist (in press)].

Eckhorn R, Bauer R, Jordan W, Brosch M, Kruse W, Munk M, Reitboeck HJ Coherent oscillations: a mechanism for feature linking in the visual cortex? Bio Cybern 1988; 60: 121-130.

Freund TF, Martin KAC, Soltesz I, Somogyi P, Whitteridge $\mathrm{D}$. Arborization pattern and postsynaptic targets of physiologically identified thalamocortical afferents in striate cortex of the macaque monkey. J Comp Neurol 1989; 289: 315-336.

Funke K, Nelle E, Li B, Wörgötter F. Corticofugal feedback improves the timing of retinogeniculate signal transmission. NeuroReport 1996; 7: 21302134.

Gerstner W, Kempter R,van Hemmen JL, Wagner H. A neuronal rule for sub-millisecond temporal coding, Nature 1996; 383, 76-78.

Gray CM, Singer W. Stimulus-specific neuronal oscillations in orientation columns of cat visual cortex. Proc Natl Acad Sci USA 1989; 86: 1698-1702.

Gray CM, McCormick DM. Chattering cells: superficial pyramidal neurons contributing to the synchronous oscillations in the visual cortex. Science 1996; 274: 109-113.

Herculano-Houzel S. Modulation of neuronal synchronous oscillations: dynamic variation with level of cortical activation and long-term use-dependent modification. Ph. D. thesis, University of Paris VI, 1999.

Houzel J-C, Milleret C, Innocenti G. Morphology of callosal axons interconnecting areas 17 and 18 of the cat. Euro J Neurosci 1994; 6: 898-917.

Innocenti GM, Lehmann P, Houzel J-C. Computational structure of visual callosal axons. Eur J Neurosci 1994; 6: 918-935.

Jeffress LA. A place theory of sound localization. J Comp Physiol 1948; 41: 35-39.

Joliot M, Ribary U, Llinas R. Neuromagnetic coherent oscillatory activity in the vicinity of $40-\mathrm{Hz}$ coexists with cognitive temporal binding in the human brain. Proc Natl Acad Sci USA 1994; 91: 11748-11751.

Laurent G, Wehr M, Davidowitz H. Temporal representations of odors in an olfactory network. J Neurosci 1996; 16: 3837-3847.

Lestienne R, Tuckwell HC, Chalansonnet M, Chaput M. Repeating triplets of spikes and oscillations in the mitral cell discharges of freely breathing rats. Eur $\mathrm{J}$ Neurosci 1999; 11: 1-9.

Lestienne R. Temporal coding with and without clocks. In: Proceedings of the Cargese Summer School 1997, Series in Mathematical Biology, Auger-Jean, World Scientific Publishing, 1999; 1-26. 
Lestienne R, Strehler B. Time structure and stimulus dependence of precisely replicating patterns present in monkey cortical neuronal spike trains. Brain Res 1987; 437: 214-238.

Lestienne R, Tuckwell HC. The significance of precisely replicating patterns in mammalian CNS spike trains. Neuroscience 1998; 82: 315-336.

MacLeod K, Laurent G. Distinct mechanisms for synchronization and temporal patterning of odor-encoding neural assemblies. Science 1996; 274: 976-979.

Makela JP., Hari R. Evidence for cortical origin of the 40 $\mathrm{Hz}$ auditory evoked response in man. Electroencephalography Clin Neurophysiol 1987; 66: 539-546.

Markram H, Lübke J, Frotscher M, Sakmann B. Regulation of synaptic efficacy by coincidence of postsynaptic APs and EPSPs. Science 1997; 275: 213-215.

Menon V, Freeman WJ, Cutillo BA, Desmond JE, Ward MF, Bressler SL, et al. Spatio-temporal correlations in human gamma band electrocorticograms. Electroencephalography Clin Neurophysiol 1996; 98: 89102.

Moiseff A, Konishi M. Neuronal and behavioral sensitivity to binaural time difference in the owl. J Neurosci 1981; 1: 40-48.

Oram MW, Wiener MC, Lestienne R, Richmond BJ. The stochastic nature of precisely timed spike patterns in visual system neuronal responses. J Neurophys 1999; 81: 3021-3033.

Roelfsema PR., Engel AK., Konig P, Singer W. Visuomotor integration is associated with zero time-lag synchronization among cortical areas. Nature 1997; 385: 157-161.

Rougeul-Buser A. Electrocortical rhythms in the $40 \mathrm{~Hz}$ band in cat: in search of their behavioural correlates, In: Buzsaki G, Llinas $R$, Singer W, Berthoz A, Christen Y, eds, Temporal Coding in the Brain, Berlin: Springer-Verlag, 1994; 103-114.

Saitoh I, Suga N. Long delay lines for ranging are created by inhibition in the inferior colliculus of the mustached bat. J Neurophysiol 1995; 74: 1-11.

Shadlen MN, Newsome MT. The variable discharge of cortical neurons: implications for connectivity, computation, and information coding. J Neurosci 1998; 18: 3870-3896.

Singer W, Gray CM. Visual feature integration and the temporal correlation hypothesis. Ann Rev Neurosci 1995; 18: 555-586.

Singer W. Synchronization of cortical activity and its putative role in information processing and learning. Ann Rev Physiol 1993; 55: 349-374.

Stopfer M, Bhagavan S, Smith BH, Laurent G. Impaired odor discrimination on desynchronization of odor-encoding neural assemblies. Nature 1997; 390: 70-74.

Strehler BL, Lestienne R. Evidence on precise time-coded symbols and memory of patterns in monkey cortical neuronal spike trains. Proc Natl Acad Sci USA, 1986; 83: 9812-9816.

Tallon-Baudry C, Bertand O, Depeuch C, Pernier J. Oscillatory gamma-band $(30-70 \mathrm{~Hz})$ activity induced by a visual search task in humans. J Neurosci 1997; 17: 722-734.

Tettoni L, Lehmann P, Houzel JC, Innocenti GM. A method for the simulation of spatiotemporal activity profiles in axonal arbors. Eur J Neurosci(Suppl) $1993 ; 6,73$.

Traub RD, Whittington MA, Colling SB, Buzsaki G, Jefferys JG. Analysis of gamma rhythms in the rat hippocampus in vitro and in vivo. $\mathrm{J}$ Physiol London 1996; 493: 471-484.

Wehr M, Laurent G. Odor encoding by temporal sequences of firing in oscillating neural assemblies. Nature 1996; 384: 162-166.

Wörgotter F, Nelle E, Li B, Funke K. The influence of corticofugal feedback on the temporal structure of visual responses of cat thalamic relay cells. J Physiol London 1998; 509: 797-815. 

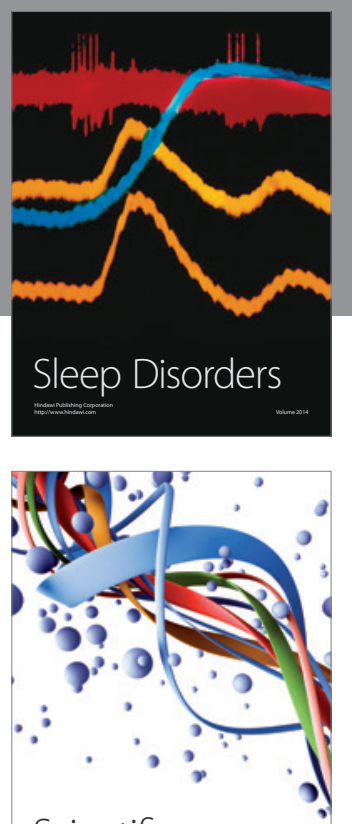

Scientifica
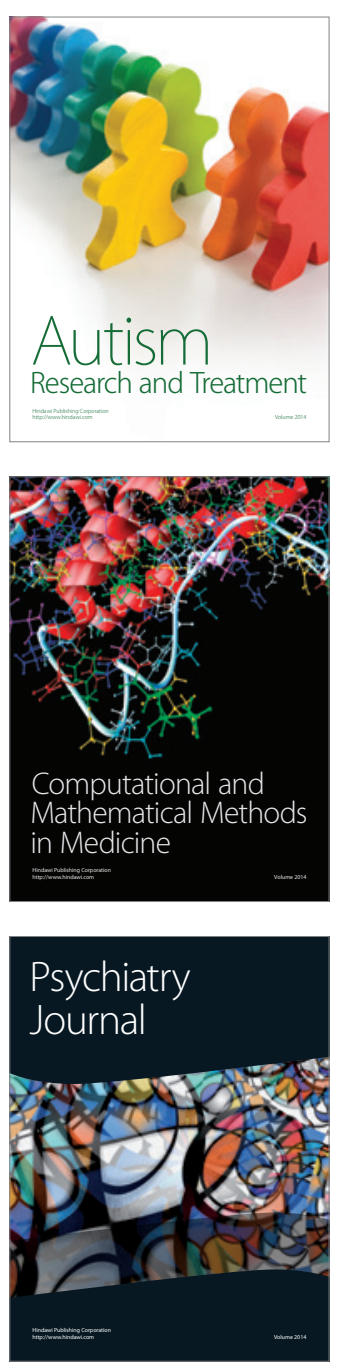
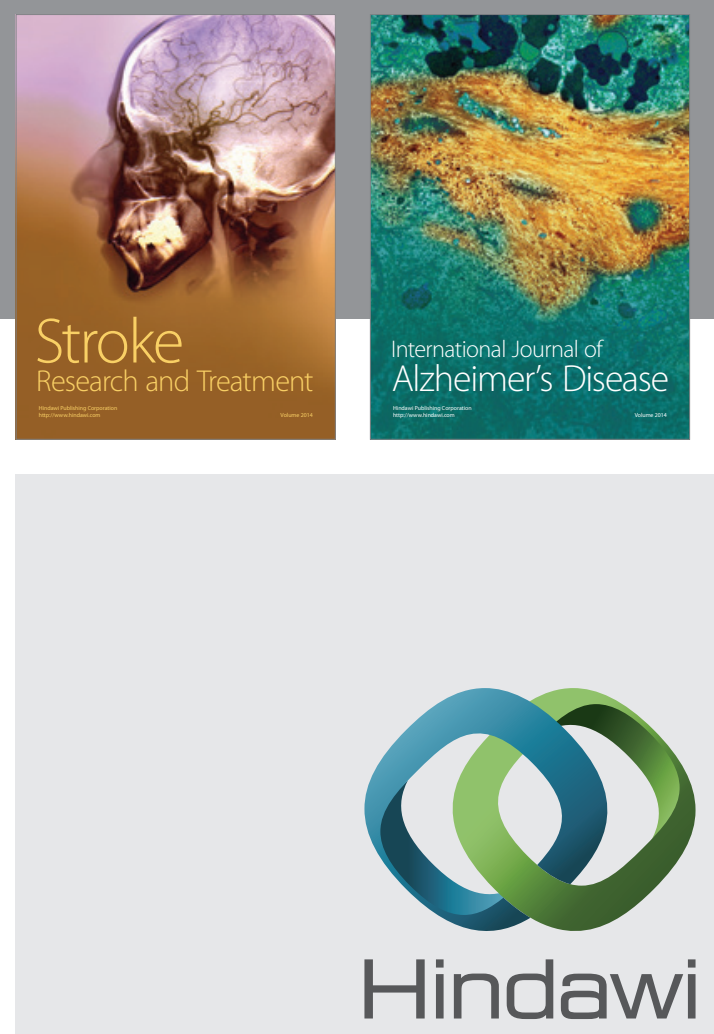

Submit your manuscripts at

http://www.hindawi.com
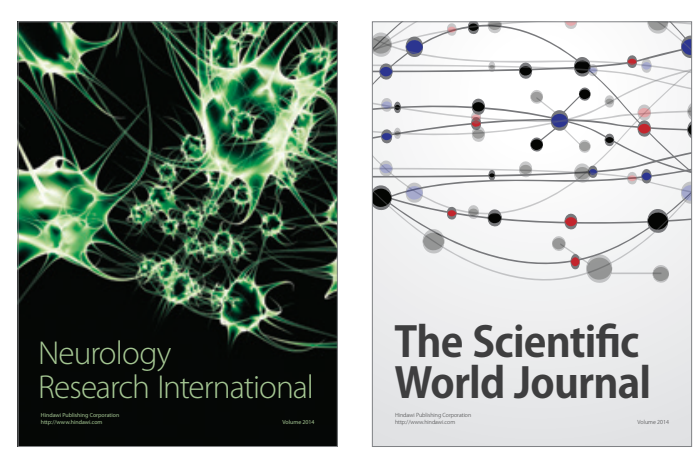

The Scientific World Journal

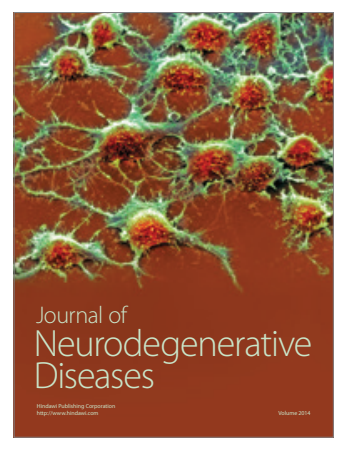

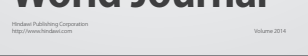

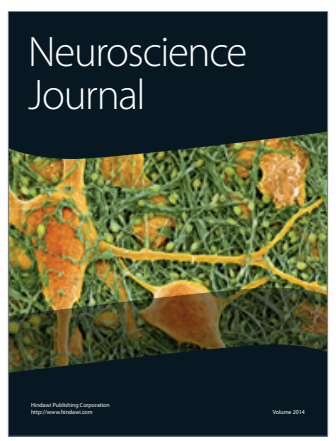

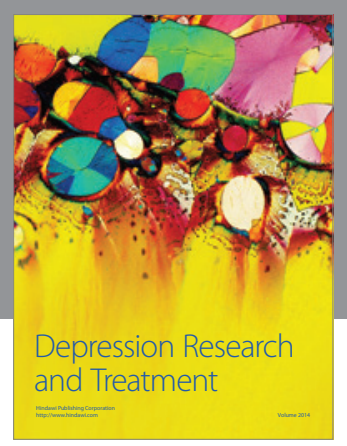
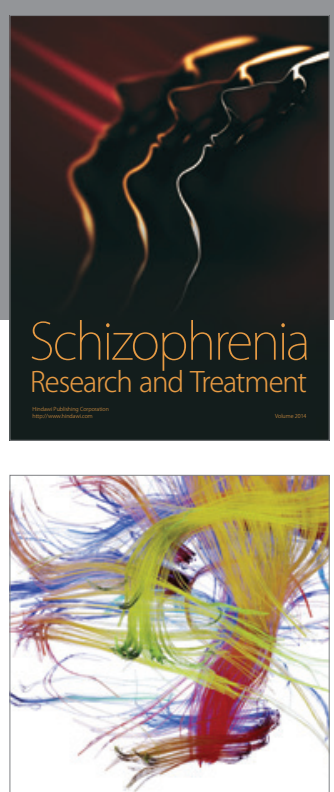

Brain Science

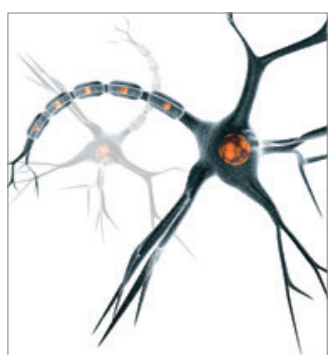

Neural Plasticity
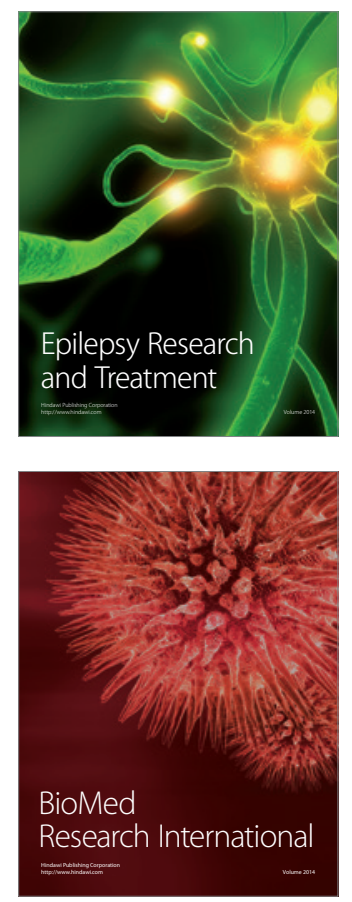

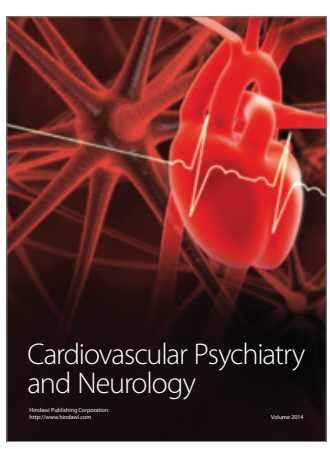

Parkinson's

Disease
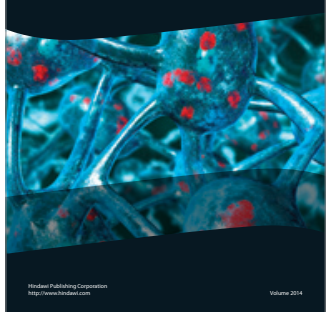